\title{
Mitgliederliste der Soncino-Gesellschaft
}

Als Grundlage der vorliegenden ersten vollständigen Liste der Mitglieder der Soncino-Gesellschaft dienen die in den vereinsinternen Publikationsorganen veröffentlichten Listen vom 15. Oktober $1924^{1}, 1$. August $1925^{2}$, 1. Februar 1927², 1. Juni $1928^{4}$ und vom Februar $1929^{5}$. Sie umfasst alle Personen, Bibliotheken, Körperschaften und Logen des U.O.B.B. ${ }^{6}$, die zu irgendeiner Zeit Mitglied der Gesellschaft waren, also auch jene, die zu einem späteren Zeitpunkt wieder ausgeschieden oder verstorben sind. Über Aufnahmegesuche und Austritte wurde in den Vereinsmitteilungen Bericht gegeben. So heißt es zum Beispiel im Bericht über die 3. Ordentliche Generalversammlung am 20. Mai 1928:

\footnotetext{
In der Zeit vom 16.4.1924 bis zum 1.5.1928 bewilligte der Vorstand die Aufnahmegesuche von 852 Mitgliedern, darunter die von 80 Bibliotheken und Körperschaften. Von diesen 852 Mitgliedern sind im Laufe der Zeit 190 wieder ausgeschieden. Somit tritt unsere Gesellschaft mit 662 Mitgliedern in das neue Geschäftsjahr ein, also eine Zunahme von 82 Mitgliedern in der letzten Berichtsperiode. ${ }^{7}$
}

Und im Bericht zur Jahresversammlung vom 29./30. April 1930 in Berlin: „In diesem Jahre schwerer wirtschaftlicher Depression war es uns leider nicht möglich, unsere Mitgliederzahl, wie wir gehofft hatten, zu vergrößern. So kommt es, daß wir auch Ende 1929 noch mit einer dicht unter der 700-Grenze liegenden Mitgliederzahl rechnen mußten.“8

1 Soncino-Gesellschaft der Freunde des jüdischen Buches e.V.: Verzeichnis der Mitglieder nach dem Stande vom 15. Oktober 1924. Berlin 1924. Die erste Mitgliederliste wurde in einer Auflage von 300 Exemplaren bei Otto von Holten gedruckt.

2 Mitgliederverzeichnis der Soncino-Gesellschaft der Freunde des jüdischen Buches. Abgeschlossen nach dem Stande vom 1. August 1925. In: Bericht über die Tätigkeit im ersten Gesellschaftsjahr. Berlin 1925.

3 Verzeichnis der Mitglieder nach dem Stande vom 1. Februar 1927. In: Soncino-Nachrichten, Beilage zu den Soncino-Blättern, Band I. Berlin 1925-1926.

4 Mitglieder-Verzeichnis der Soncino-Gesellschaft der Freunde des jüdischen Buches nach dem Stande vom 1. Juni 1928. In: Mitteilungen der Soncino-Gesellschaft 3 (Juli 1928).

5 Nachtrag zum Mitgliederverzeichnis vom 1. Juni 1928. Neuaufnahmen bis zum 20. Februar 1929. In: Mitteilungen der Soncino-Gesellschaft 4 (Februar 1929).

6 Unabhängiger Orden Bne Briss (U.O.B.B.) gegründet 1843 als geheime Loge.

7 Bericht über die 3. Ordentliche Generalversammlung am 20. 05.1928. In: Mitteilungen der Soncino-Gesellschaft 3 (Juli 1928), S. 1f.

8 Bericht über die Jahresversammlung der Soncino-Gesellschaft am 29. und 30. April 1930 in Berlin. In: Mitteilungen der Soncino-Gesellschaft 5 (August 1930), S. 3. 
ANTWERPEN

Salomon Fink, 43 Avenue Golmaere, Antwerpen

BALTIMORE

Prof.Dr.D.S.Blondheim, John Hopkins Univ., Baltimore M.D., U.S.A.

BRƯSSEL

Oskar Jurovics, Brüssel, 25 Rue Berkmans

BERLIN

Josef Altmann, Bln=Schlachtensee, Luisenstr. 6 8

Raja Arinstein, Bln=Wilmersdorf, Konstanzer Str.53 116

Dr.med.Willy Auerbach, Berlin W 50, Fürther Str.11 a 62

Leo Bäcker, Berlin W9, Potsdamer Str. $20 \quad 30$

Rabb.Dr.L.Baeck, Berlin W62, Burggrafenstr.19 48

Direktor Karl M.Baer, Berlin W62, Kleiststr.11 33

Dr.jur.Aron Barth, Berlin NW7, Sommerstr.5 80

Dr. Ruben Beatus, Bln=Neukölln, Donaustr. 129

b.Wilszinski

169

Werner Behr, Berlin N 54, Alte Schönhauser Str.30 17

Alfred Berger, Berlin W62, Bülowstr.16 64

Regierungsrat Dr.jur.Ludw. Berliner, Bln=Wilmers= dorf, Holsteinische Str. 25

Jakob Blumenthal, Bln=Charlottenburg, Uhlandstr.30 176

San.:Rat Dr.Gustav Bradt, Berlin W35, Potsdamer

Str. 27

Rudolf Braude, Bln $\times$ Wilmersdorf, Prinzregentenstr. 8752

Frl.Emmy Broido, Berlin W10, Kaiserin=Augusta=

Str.73 (b.Dr.Weiß)

Lipa Bronstein, Bln=Charlottenburg, Dahlmannstr. 8147

Martin Brunn, Bln=Wilmersdorf, Trautenaustr. 8179
Abb. 40: Auszug aus der ersten Mitgliederliste der Soncino-Gesellschaft vom 15. Oktober 1924.

Nach Auswertung der vorliegenden Listen bewilligte der Vorstand die Aufnahme von 884 Mitgliedern, darunter 792 Personen, 55 Bibliotheken und Körperschaften sowie 37 Logen des U.O.B.B. Die höchste Mitgliederzahl verzeichnet Deutschland mit 651 Personen, gefolgt von Österreich mit 32 und Polen mit 19. Der bei weitem größte Teil der Mitglieder stammte aus Berlin mit 304 Personen und 9 Bibliotheken. Unter den Städten insgesamt folgen Frankfurt/Main mit 40 Personen, Hamburg mit 33 und Wien mit 30.

Ausgehend von den in den Listen verzeichneten Berufsgruppen (soweit angegeben) dominieren die Rechtsanwälte mit 79 Mitgliedern (inklusive vier Justizräte und fünf Syndici), davon 26 in Berlin; gefolgt von 64 Ärzten (Dr. med. und zwei Prof. Dr. med.), darunter eine Kinderärztin; des Weiteren 26 Direktoren (zuzüglich 
drei Bankdirektoren und zwei Generaldirektoren) und 24 Rabbiner (darunter ein Landesrabbiner und ein Stadt- und Bezirksrabbiner). Bei den Logen des U.O.B.B. dominiert ebenfalls Deutschland mit 28 Mitgliedern.

Unter den Mitgliedern befinden sich auch 21 Frauen, darunter die Berliner Zionistin Lina Wagner-Tauber (Nr. 689), Mitbegründerin der ersten nationaljüdischen Frauenorganisation in Deutschland; die Kölner Kinderärztin Alice Schiff (Nr. 423); die Rezitatorin Edith Herrnstadt-Oettingen (Nr. 152) aus Nowawes bei Potsdam sowie die Schriftstellerin Lilly de Jong (Nr. 70) aus Berlin. ${ }^{9}$

Die Liste weist zudem 13 nichtjüdische Personen und Institutionen als außerordentliche Mitglieder (a.o.) auf, darunter sechs Bibliotheken: die Deutsche Bücherei, Bayerische Staatsbibliothek, Stadtbibliothek Frankfurt/Main, Universitätsbibliothek Berlin, Orientalische Abteilung der Preußischen Staatsbibliothek Berlin, Bibliothek der Deutschen Morgenländischen Gesellschaft ebenso die Leipziger Offizin W. Drugulin. Unter den Personen befinden sich der Buchwissenschaftler und Papierhistoriker Hans H. Bockwitz (Nr. a.o. 470), der Verleger Richard Hadl (Nr. a.o. 187), der 1926 die gleichnamige Offizin in Leipzig gründete, und der Leipziger Antiquar und Verleger Karl W. Hiersemann (Nr. a.o. 186).

Im Folgenden soll auf einige wesentliche Probleme hingewiesen werden, die bei der Auswertung der Listen zutage traten.

\section{Mitgliedsnummern bis 800}

Die Zählung bis zur Mitgliedsnummer 800 enthält 31 nicht vergebene Nummern (269, 272, 279, 331, 358, 373, 397, 450, 454, 498, 544, 549, 556, 565, 572, 579, 582, 593, 594, 597, 598, 610, 626, 627, 633, 637, 696, 705, 780, 783, 799).

Bei der Zählung treten Verwechslungen von Namen und Mitgliedsnummern auf, verstärkt in den Nummern 14 bis 20. Als Beispiel seien hier die Zionisten Gotthold Weil und Siegmund Kaznelson erwähnt, die in den verschiedenen Listen wechselnd mit der Nummer 71 oder 72 erscheinen. Hier, wie in allen anderen Fällen, wurde für die Namensnennung die jeweilige Angabe 71 | 72 festgelegt.

9 Raja Arinstein (Berlin), Amalie Berger (Berlin), Emmy Broido (Berlin), Nelly Frank (Berlin), Hedwig Gutman (München), Frieda Hichenberg (Nürnberg), Elsbeth Hirschmann (Köln), Martha Katz (Witten), Ida Marx (Berlin), Grete Meyer (Berlin), Rosi Ester Peiser (Berlin), Vera Sasson (Schottland), Irma Schindler (Hamburg), Emmi Simon (Berlin), Rahel Wolf (Königsberg), Helene Wolff (Berlin), Gertrud Wunderlich (Berlin). 
Darüber hinaus treten Doppelungen von Nummern auf. Diese lassen sich möglicherweise mit dem Ausscheiden oder Tod eines Mitglieds erklären, wobei die Nummer dann an ein anderes Mitglied oder eine Bibliothek/Körperschaft übertragen wurde. Nachvollziehbar erscheint dies am Beispiel des Gründungsmitglieds Siegfried Wolff, nach dessen Tod 1926 seine Frau Helene die Mitgliedschaft mit der Nummer 4 weiterführte.

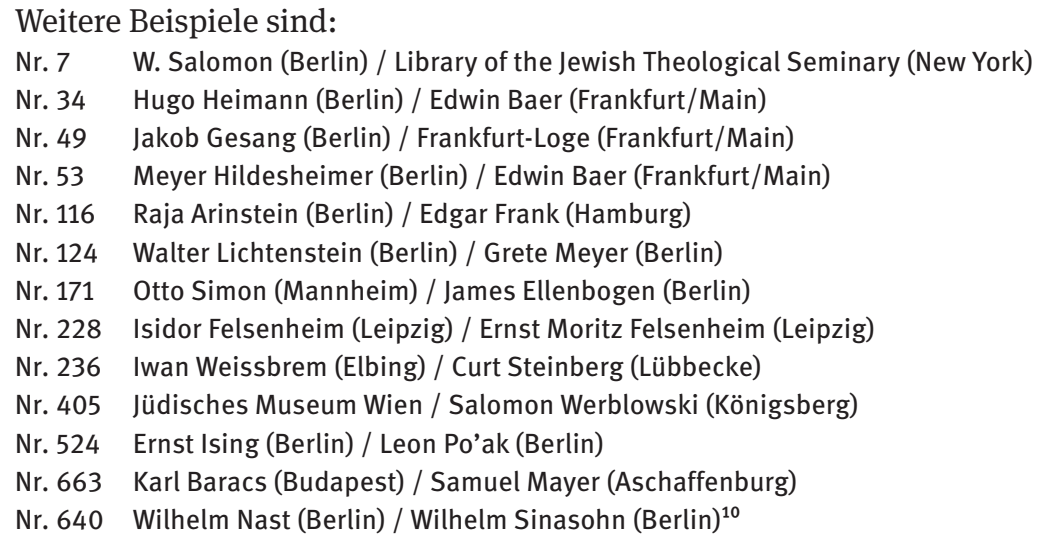

Daneben gibt es das Beispiel von Ernst Eisler, Teplitzsch-Schönau, der 1924 und 1925 die Mitgliedsnummer 221 hatte, 1927 keine namentliche Erwähnung findet und 1928 mit der Nummer 788 unter der gleichen Adresse erneut erscheint.

\section{Zählung mit Doppelnummern}

In der Liste vom Juni 1928 sind 54 Mitglieder erwähnt, die eine Doppelnummer haben, darunter die Zionisten Martin Buber (Nr. 52/221), Ludwig Mayer (Nr. 43/177), Franz Rosenzweig (Nr. 51/220), Chaim Weizmann (Nr. 36/165) ebenso das Hebrew Union College, Cincinnati (Nr. 41/175), die Staats- und Universitätsbibliothek Hamburg (Nr. 35/162) und die Universitäts-Bibliothek Leipzig (Nr. 25/124). Die beiden Letzteren müssten eigentlich als nichtjüdische Körperschaften als außerordentliche Mitglieder aufgeführt sein. Generell fehlen für diese Nummernzählung bislang weitere Informationen.

10 Beide Mitglieder firmieren unter derselben Postanschrift. 


\section{Keine Nummernzählung}

Die letzte Mitgliederliste, die die Neuaufnahmen bis zum 20. Februar 1929 erfasst, weist 45 Einträge ohne Nummerierung auf. Problematisch sind dabei die Beispiele von Bankdirektor Gustav Benario, der in den Listen von 1925 bis 1928 als Frankfurter Mitglied die Nummer 412 innehat und 1929 als Neuzugang in Berlin ohne Nummer erscheint. Das gleiche lässt sich für den Schriftsteller Chaim Nachman Bialik, Tel Aviv, feststellen, der in den beiden ersten Mitgliederlisten 1924/25 die Nummer 93 hat, 1927/28 nicht erwähnt ist und 1929 als Neuzugang verzeichnet wird.

Im Fall der Nummerndopplung von Rudolf Schick ließen sich zwei verschiedene Personen identifizieren: Zum einen der Inhaber der gleichnamigen Leipziger Verlagsbuchhandlung mit der Nummer 127, der nach Kanada emigrierte, sowie der in der letzten Liste ohne Nummer aufgeführte, gebürtige Prager Rudolf Schick, der im Ghetto Lodz ermordet wurde.

Problematisch erscheint eine genau Identifizierung von Heinrich Cohn, Tel Aviv (Nr. 673), bei dem es sich um den Bankier und späteren Mitbegründer von Naharija handeln könnte oder um Heinrich Cohn aus Jerusalem (o. Nr.).

Schließlich finden sich in verschiedenen Materialien Namen von Mitgliedern, die in keiner der bislang aufgefundenen Mitgliederlisten aufgeführt sind. Hierzu zählen Siegfried Nossbaum (Nahalal/Palästina) ${ }^{11}$, Dr. Hans Lewenz, der im Druckvermerk zur 103. Publikation der Soncino-Gesellschaft erwähnt wird, ${ }^{12}$ Fritz Bamberger, der den Posten des Schriftführers von Herrmann Meyer übernommen hatte, und Richard Beer-Hofmann, der Mitglied des ersten Ehrenausschusses war.

11 Josef Altmann an Heinrich Loewe, 15.02.1934, Beit Ariela (BA), Boxnr. 7.

12 Vgl. die Bibliographie im Anhang. 
Die Auflistung der Mitglieder nach Orten und Ländern sowie Institutionen folgt der Vorlage in den Mitgliederlisten.

\section{Deutschland}

\begin{tabular}{|c|c|c|c|c|c|}
\hline Bütow & Hugo & & & Allenstein & 785 \\
\hline Krüger & Heinrich & Dr. med. & & Allenstein & 571 \\
\hline Lesser & Alfred & & Rechtsanwalt & Allenstein & 280 \\
\hline Romm & Max & Dr. med. & & Allenstein & 578 \\
\hline Segall & Leo & & & Allenstein & 624 \\
\hline Wistinetzki & Leo & & Rechtsanwalt & Allenstein & 408 \\
\hline Wolffheim & Heinrich & Dr. med. & & Allenstein & 447 \\
\hline Rothschild & Richard & Dr. med. & & $\begin{array}{l}\text { Alzey/ } \\
\text { Braunschweig }\end{array}$ & 305 \\
\hline Mayer & Samuel & & & Aschaffenburg & 663 \\
\hline Weil & Siegfried & Dipl.-Ing. & & Augsburg & 636 \\
\hline Appel & Richard & Dr. med. & & Bad Oeynhausen & 666 \\
\hline Roos & Fritz & & & Barmen & o. Nr. \\
\hline Abrahamson & Martin & & & Berlin & 573 \\
\hline Abramczyk & W. Willy & & & Berlin & 762 \\
\hline Alexander & Alfred & Dr. med. & & Berlin & 309 \\
\hline Altmann & Josef & & & Berlin & 8 \\
\hline Altmann & Kurt & & & Berlin & 676 \\
\hline Altschul & Jewsei & Dr. & & Berlin & 732 \\
\hline Alweiß & Siegfried & & & Berlin & 683 \\
\hline Anders & Alfred & & & Berlin & $30 / 146$ \\
\hline Anfänger & Lutz & Dr. med. & & Berlin & o. Nr. \\
\hline Arinstein & Raja & & & Berlin & 116 \\
\hline Arndt & Isidor & & & Berlin & 383 \\
\hline Auerbach & Willy & Dr. med. & & Berlin & 62 \\
\hline Badt & Hermann & Dr. jur. & Ministerialdirektor & Berlin & 656 \\
\hline Baeck & Leo & Dr. & Rabbiner & Berlin & 48 \\
\hline Bäcker & Leo & & & Berlin & 30 \\
\hline Baer & Karl M. & & Direktor & Berlin & 33 \\
\hline Barth & Aron & Dr. jur. & Rechtsanwalt & Berlin & 80 \\
\hline Baruch & Lippmann & Dr. med. & & Berlin & 569 \\
\hline Battsek & Kurt & & Syndikus & Berlin & 389 \\
\hline
\end{tabular}




\begin{tabular}{|c|c|c|c|c|c|}
\hline Beatus & Ruben & Dr. & & $\begin{array}{l}\text { Berlin/Homburg/ } \\
\text { Frankfurt a.M. }\end{array}$ & 169 \\
\hline Behr & Werner & & & Berlin & $15 \mid 17$ \\
\hline Bendix & Emil & & & Berlin & 726 \\
\hline Benedik & Paul & & & Berlin & 684 \\
\hline Beradt & Martin & Dr. jur. & Rechtsanwalt & Berlin & 411 \\
\hline Berger & Alfred & & & Berlin & 64 \\
\hline Berger & Amalie & & & Berlin & $4 / 31$ \\
\hline Berger & Hermann & & & Berlin & 769 \\
\hline Bergmann & Siegfried & & Rechtsanwalt & Berlin & 740 \\
\hline Berkmann & Josef & & & Berlin & 308 \\
\hline Berliner & Ludwig & Dr. jur. & Regierungsrat & Berlin & 181 \\
\hline Bernhard & Georg & Prof. & Chefredakteur & Berlin & $7 / 52$ \\
\hline Bier & Norbert & & & Berlin & 580 \\
\hline Bier & Siegfried F. & & & Berlin & 581 \\
\hline Bloch & Richard & & $\begin{array}{l}\text { Regierungsbau- } \\
\text { meister }\end{array}$ & Berlin & 367 \\
\hline Blumenthal & Jakob & & & Berlin & 176 \\
\hline Boas & Bruno & & & Berlin & 455 \\
\hline Bochenek & Berthold & & Direktor & Berlin & 731 \\
\hline Boehm & Erich & & Rechtsanwalt & Berlin & 743 \\
\hline Bondy & Paul & & & Berlin & 349 \\
\hline Bradt & Gustav & Dr. & Sanitätsrat & Berlin & 77 \\
\hline Brandus & Martin & & & Berlin & 390 \\
\hline Braude & Rudolf & & & Berlin & 52 \\
\hline Braun & A. M. & & & Berlin & 328 \\
\hline Braun & Siegmund & & & Berlin & 329 \\
\hline Braun & Simon & & & Berlin & 330 \\
\hline Broido & Emmy & & & Berlin & 59 \\
\hline Bronstein & Lipa & & & Berlin & 147 \\
\hline Brunn & Martin & & & Berlin & 179 \\
\hline Butten(r)wieser & Samson & & & Berlin & 38 \\
\hline Cahn & Victor & & & Berlin & 391 \\
\hline Casparius & Edgar & & & Berlin & 661 \\
\hline Caspary & Eugen & & & Berlin & 639 \\
\hline Choyke & Fritz & & & Berlin & 502 \\
\hline Cohn & Berthold & Dr. jur. & Syndikus & Berlin & 503 \\
\hline
\end{tabular}




\begin{tabular}{|c|c|c|c|c|c|}
\hline Cohn & Conrad & & & Berlin & 560 \\
\hline Cohn & Erich & & Rechtsanwalt & Berlin & 26 \\
\hline Cohn & Harry & Dr. & & Berlin & 31 \\
\hline Cohn & Leonhard & Dr. & Landgerichtsrat & Berlin & 312 \\
\hline Cohn & Otto & & & Berlin & 714 \\
\hline David & Willi & & Direktor & Berlin & $14 \mid 15$ \\
\hline Diamant & B. & Dr. med. & & Berlin & 536 \\
\hline Diamant & David & & & Berlin & 39 \\
\hline Ehrlich & Alexander & & & Berlin & 194 \\
\hline Eisner & Fritz & Dr. rer. pol. & & Berlin & 217 \\
\hline Eitingon & Max & Dr. med. & & Berlin & 505 \\
\hline Eitingon & Michael & & & Berlin & 678 \\
\hline Eliasberg & Ahron & Dr. phil. & Weltverlag & Berlin & 32 \\
\hline Ellenbogen & James & Dr. & & Berlin & 171 \\
\hline Engel & Leo & & & Berlin & o. Nr. \\
\hline Engel & Siegfried & & & Berlin & 36 \\
\hline Feibusch & Aron & & & Berlin & 51 \\
\hline Feld & Hans & Dr. jur. & & Berlin & $16 \mid 18$ \\
\hline Feuerring & Isaak & & & Berlin & 796 \\
\hline Fraenkel & Max & & & Berlin & 136 \\
\hline Fränkel & S. Martin & & & Berlin & 189 \\
\hline Frank & Alfred & & & Berlin & 631 \\
\hline Frank & Nelly & & & Berlin & 766 \\
\hline Freimann & Martin & & & Berlin & 40 \\
\hline Freud & Ernst & Dipl.-Ing. & & Berlin & 196 \\
\hline Freund & Ismar & & & Berlin & 19 \\
\hline Freyer & Kurt & Dr. phil. & & Berlin & $17 \mid 19$ \\
\hline Friedeberger & Paul & Dr. & & Berlin & o. Nr. \\
\hline Gans & Sally & Dr. & Rabbiner & Berlin & 570 \\
\hline Garbáty & Moritz & Dr. & & Berlin & $651 \mid 661$ \\
\hline Gesang & Jakob & & & Berlin & 49 \\
\hline Glaser & Siegfried & & & Berlin & 263 \\
\hline Goeritz & Erich & & & Berlin & 115 \\
\hline Goldberg & Chaim & & & Berlin & 274 \\
\hline Goldkraut & Hermann & & & Berlin & 692 \\
\hline Goldmann & Nachum & Dr. & & Berlin & 65 \\
\hline Goldschmidt & Karl & Dr. jur. & Rechtsanwalt & Berlin & 753 \\
\hline
\end{tabular}




\begin{tabular}{|c|c|c|c|c|c|}
\hline Goslar & Hans & & $\begin{array}{l}\text { Oberregierungsrat/ } \\
\text { Ministerialrat }\end{array}$ & Berlin & 254 \\
\hline Gradenwitz & Rafael & & & Berlin & 37 \\
\hline Graetz & Martin & Dr. med. & & Berlin & 567 \\
\hline Graßmann & Karl & Dr. jur. & Rechtsanwalt & Berlin & 193 \\
\hline Graupe & Paul & & & Berlin & 511 \\
\hline Gronemann & Sammy & & Rechtsanwalt & Berlin & 10 \\
\hline Grüngart & Faywel & & Direktor & Berlin & 733 \\
\hline Gusowski & J. & & & Berlin & 741 \\
\hline Halberstadt & James & & & Berlin & 725 \\
\hline Hartbrodt & Alfred & & & Berlin & 182 \\
\hline Heimann & Hugo & Dr. phil. & & Berlin & 34 \\
\hline Heller & Josua & Dr. & & Berlin & 54 \\
\hline Henschel & Max & & & Berlin & 256 \\
\hline Herlitz & Georg & Dr. phil. & & Berlin & 69 \\
\hline Herzog & Martin & & Gerichtsassessor & Berlin & 514 \\
\hline Heskel & Leo & & & Berlin & 265 \\
\hline Hildesheimer & Meier & Dr. & Rabbiner & Berlin & 53 \\
\hline Hildesheimer & Schalom & Dr. med. & & Berlin & 722 \\
\hline Hirsch & Josepf & & & Berlin & $38 / 171$ \\
\hline Hirsch & Siegfried & & Direktor & Berlin & 761 \\
\hline Hirschfeld & Leo & Dr. & Amtsrichter & Berlin & 421 \\
\hline Hirschfeld & Robert & & Lehrer & Berlin & 42 \\
\hline Hollander & Gottfried & Dr. & Rechtsanwalt & Berlin & 426 \\
\hline Horodisch & Abraham & Dr. rer. pol. & & Berlin & 3 \\
\hline Horodisch & Samuel & & & Berlin & 97 \\
\hline Ising & Ernst & Dr. & & Berlin & 524 \\
\hline Israel & Wilfrid & & & Berlin & o. Nr. \\
\hline Ittmann & Julius & & Rechtsanwalt & Berlin & $102 \mid 103$ \\
\hline Jacobus & Adolf & Dr. & Rabbiner & Berlin & o. Nr. \\
\hline Japhet & Jakob & & & Berlin & 518 \\
\hline Jastrow & Josef & & & Berlin & 460 \\
\hline Joachim & Günther & & & Berlin & 318 \\
\hline Jolles & Oskar & Dr. & Direktor & Berlin & 128 \\
\hline Jolowicz & Albert & & & Berlin & 712 \\
\hline Jong, de & Lilly & & & Berlin & 70 \\
\hline Joseph & Louis & & & Berlin & 745 \\
\hline
\end{tabular}




\begin{tabular}{|c|c|c|c|c|c|}
\hline Joseph & Wilhelm & & & Berlin & 742 \\
\hline Jungmann & Max & Dr. med. & & Berlin & 323 \\
\hline Jurovics & Samuel & & & Berlin & $23 \mid 29$ \\
\hline Kahan & B. & & & Berlin & 519 \\
\hline Kahn & Fritz & Dr. med. & & Berlin & 29 \\
\hline Kaminski & Paul & & & Berlin & 198 \\
\hline Kander & Adolf & & & Berlin & 310 \\
\hline Karger & Siegbert & & & Berlin & 247 \\
\hline Kastan & J. & Dr. med. & & Berlin & 340 \\
\hline Kaznelson & Siegmund & Dr. & & Berlin & $71 \mid 72$ \\
\hline Kirschner & Bruno & Dr. phil. & & Berlin & $18 \mid 20$ \\
\hline Kirschstein & Sally & & & Berlin & 422 \\
\hline Klee & Alfred & Dr. jur. & Rechtsanwalt & Berlin & 83 \\
\hline Klein & Isi & & & Berlin & 794 \\
\hline Knoller & Simon $M$. & & & Berlin & 61 \\
\hline Kober & Max & & & Berlin & 66 \\
\hline Kober & Max W. & & & Berlin & o. Nr. \\
\hline Kochmann & Hans $\mathrm{H}$. & & & Berlin & 369 \\
\hline Kohn & Louis A. & & & Berlin & 183 \\
\hline Kollenscher & Max & Dr. & Rechtsanwalt & Berlin & 748 \\
\hline Kronheim & Max & & & Berlin & 757 \\
\hline Kuttner & Leo & & & Berlin & 754 \\
\hline Labisch & Richard & & & Berlin & 677 \\
\hline Lachmann & Rafael & & & Berlin & 41 \\
\hline Lamm & Fritz & Dr. & Rechtsanwalt & Berlin & 435 \\
\hline Lamm & Louis & & & Berlin & 16 \\
\hline Landau & J. & & Chefredakteur & Berlin & 220 \\
\hline Landsberg & Alfred & Dr. jur. & Rechtsanwalt & Berlin/Wiesbaden & 233 \\
\hline Laske & Gotthard & & & Berlin & 109 \\
\hline Lehmann & Arthur & & & Berlin & 706 \\
\hline Lehr & Philipp & & & Berlin & 755 \\
\hline Leszynsky & Eduard & Dr. & Rechtsanwalt & Berlin & 595 \\
\hline Leszynsky & Rudolf & Dr. & Direktor & Berlin & 209 \\
\hline Levy & Albert & & & Berlin & 301 \\
\hline Levy & Arthur & Dr. & Rabbiner & Berlin & 326 \\
\hline Levy & Bernhard & & Lehrer & Berlin & 101 \\
\hline Levy & Moritz & & & Berlin & 35 \\
\hline
\end{tabular}




\begin{tabular}{|c|c|c|c|c|c|}
\hline Lewin & Karl & Prof. Dr. med. & & Berlin & 548 \\
\hline Lewit & Max & & & Berlin & o. Nr. \\
\hline Lewy & Max & Dr.-Ing. & & Berlin & 117 \\
\hline Lichtenstein & Walter & & & Berlin & 124 \\
\hline Lion & Paul & Dr. jur. & Rechtsanwalt & Berlin & 21 \\
\hline Lipschitz & Georg & & Direktor & Berlin & 211 \\
\hline Litthauer & Carl & & & Berlin & 253 \\
\hline Loeb & Leopold A. & Dr. rer. pol. & & Berlin & 445 \\
\hline Loebenstein & Karl & & & Berlin & 102 \\
\hline Loewe & Heinrich & Prof. Dr. & & Berlin & 6 \\
\hline Loewe & Martin & Dr. & & Berlin & 752 \\
\hline Loewenthal & Abraham & Dr. & Rabbiner & Berlin & 89 \\
\hline Lomnitz & Arthur & & Rechtsanwalt & Berlin & 618 \\
\hline Lux & Bruno & Dr. med. & & Berlin & 216 \\
\hline Marcus & Alfred & Dr. & & Berlin & 58 \\
\hline Marx & Ida & & & Berlin & 500 \\
\hline Marx & Moses & & & Berlin/Cincinnati & 2 \\
\hline Mass & Ruben & & & Berlin & 392 \\
\hline Matzdorf & Georg & & & Berlin & 177 \\
\hline Mayer & Ludwig & & & Berlin & $43 / 177$ \\
\hline Mayer & Max & Dr. & Assessor & Berlin & 370 \\
\hline Mayer & Richard & & & Berlin & 721 \\
\hline Meisl & Josef & Dr. phil. & & Berlin & 5 \\
\hline Mendelsohn & Jakob & & & Berlin & 50 \\
\hline Mendelsohn & Moses & & & Berlin & o. Nr. \\
\hline Mendelsohn & Oskar & & & Berlin & 255 \\
\hline Merzbach & Siegmund & & & Berlin & $44 / 180$ \\
\hline Meyer & Adolf & & & Berlin & 717 \\
\hline Meyer & Fritz & & & Berlin & 371 \\
\hline Meyer & Grete & & & Berlin & 124 \\
\hline Meyer & Herrmann & & & Berlin & 1 \\
\hline Meyersohn & Herbert & Dr. med. & & Berlin & 268 \\
\hline Michael & Jakob & & & Berlin & o. Nr. \\
\hline Michael & Max & Dr. & & Berlin & 88 \\
\hline Michaelis & Walter & & Amtsgerichtsrat & Berlin & 341 \\
\hline Michel & Erwin & Dr. & Syndikus & Berlin & 427 \\
\hline Mittwoch & Eugen & Prof. Dr. phil. & & Berlin & 81 \\
\hline
\end{tabular}




\begin{tabular}{|c|c|c|c|c|c|}
\hline Mittwoch & Felix & Dr. jur. & Rechtsanwalt & Berlin & 685 \\
\hline Mosert & Fritz & & & Berlin & 139 \\
\hline Mosessohn & Emil & & & Berlin & 200 \\
\hline Munter & Curt & & Direktor & Berlin & 197 \\
\hline Nast & Wilhelm & & & Berlin & 640 \\
\hline Nothmann & Hugo & Dr. med. & & Berlin & $3 / 27$ \\
\hline Peiser & Rosi Ester & Frau Dr. & & Berlin & 526 \\
\hline Pessen & Eugen & Dr. & & Berlin & 475 \\
\hline Pinczower & Ephraim & Dr. med. & & Berlin & 56 \\
\hline Pinner & Eugen & Dr. & & Berlin & 715 \\
\hline Pinner & Sally & & Rechtsanwalt & Berlin & 724 \\
\hline Platz & Alfred & Dr. jur. & Rechtsanwalt & Berlin & 287 \\
\hline Pleßner & Jakob & & & Berlin & 707 \\
\hline Po'ak & Leon & Dr. & & Berlin & 524 \\
\hline Posen & Leo & & & Berlin & 746 \\
\hline Posner & Richard & & & Berlin & 22 \\
\hline Posner & Kurt & & & Berlin & 675 \\
\hline Rabau & Alfred & Dr. jur. & Rechtsanwalt & Berlin & 55 \\
\hline Raschkes & Benjamin & & & Berlin & 311 \\
\hline Rau & Arthur & & Assessor & Berlin & 85 \\
\hline Rawack & Wolfgang & & & Berlin & o. Nr. \\
\hline Rosenbaum & Hermann & & & Berlin & 601 \\
\hline Rosenbaum & Kurt & & & Berlin & 547 \\
\hline Rosenberg & Heinrich & & & Berlin & o. Nr. \\
\hline Rosenberg, von & G. J. & Dr. phil. & Hofrat & Berlin & 160 \\
\hline Rosenblatt & Leo & & & Berlin & 44 \\
\hline Rosenfeld & Leo & & Direktor & Berlin & o. Nr. \\
\hline Rosenstein & Werner & & & Berlin & 758 \\
\hline Rosenthal & Karl & Pred. Dr. & & Berlin & 737 \\
\hline Rosenthal & Willy & Dr. & & Berlin & 121 \\
\hline Roth & Walter & & & Berlin & 232 \\
\hline Ruhs & Adolf & & & Berlin & 299 \\
\hline Sachs & Arthur & Dr. & & Berlin & 750 \\
\hline Sachs & Hans & Dr. & & Berlin & 418 \\
\hline Salomon & Emil & Dr. med. & & Berlin & $8 / 57$ \\
\hline Salomon & Leo & & Direktor & Berlin & 118 \\
\hline Salomon & w. & Prof. Dr. & & Berlin & 7 \\
\hline
\end{tabular}




\begin{tabular}{|c|c|c|c|c|c|}
\hline Sänger & Jakob & & & Berlin & 529 \\
\hline Schachtel & & & $\begin{array}{l}\text { Justizrat/ } \\
\text { Rechtsanwalt }\end{array}$ & Berlin & 144 \\
\hline Schatz & Ludwig & Dipl.-Ing & Direktor & Berlin & 686 \\
\hline Scherbel & Fritz & Dr. & & Berlin & 277 \\
\hline Schiesser & Bruno & & & Berlin & 284 \\
\hline Schiller & Max & & & Berlin & 368 \\
\hline Schmidt & Max & & & Berlin & 555 \\
\hline Schmidt & Sally & & & Berlin & 566 \\
\hline Schneider & Richard & & & Berlin & 644 \\
\hline Schneider & Lambert & & & Berlin & o. Nr. \\
\hline Schnelling & Moritz & & & Berlin & 723 \\
\hline Scholem & Erich & & & Berlin & 659 \\
\hline Scholem & Reinhold & & & Berlin & 699 \\
\hline Schönberg & Karl & Dr. & Rechtsanwalt & Berlin & 735 \\
\hline Schottländer & Erich & Dr. med. & & $\begin{array}{l}\text { Berlin/Mannheim/ } \\
\text { Ludwigshafen }\end{array}$ & $/ 155$ \\
\hline Schragenheim & Bernhard & & & Berlin & 45 \\
\hline Schragenheim & Erich & & & Berlin & 46 \\
\hline Schreyer & Georg & & & Berlin & 621 \\
\hline Schwarz & Julius & & & Berlin & $17 / 98$ \\
\hline Schwarz & Karl & Dr. & & Berlin & o. Nr. \\
\hline Schwarz & Ludwig & & & Berlin & 393 \\
\hline Schweitzer & Ernst & Dr. & & Berlin & 697 \\
\hline Seidmann & Jankew & & & Berlin & 195 \\
\hline Selbiger & Heinrich & & & Berlin & 260 \\
\hline Silberblatt & M. & & & Berlin & 114 \\
\hline Silberstrom & Leo & Dr. phil. & & Berlin & 173 \\
\hline Simon & Emmi & & & Berlin & 264 \\
\hline Simon & Fritz & Dr. jur. & Rechtsanwalt & Berlin & 67 \\
\hline Simon & Moritz & & Direktor & Berlin & 11 \\
\hline Sinasohn & Erhard & & & Berlin & 286 \\
\hline Sinasohn & Max & & & Berlin & 444 \\
\hline Sinasohn & Wilhelm & & & Berlin & 640 \\
\hline Sobernheim & Moritz & Prof. Dr. & Legationsrat & Berlin & 73 \\
\hline Spanier & Arthur & Dr. phil. & & Berlin & 13 \\
\hline Speyer & Siegfried & Dr. & & Berlin & 727 \\
\hline
\end{tabular}




\begin{tabular}{|c|c|c|c|c|c|}
\hline Stahl & Hermann & Dr. med. & & Berlin & 185 \\
\hline Stein & Arthur & Dr. & Rechtsanwalt & Berlin & o. Nr. \\
\hline Steinhardt & Jakob & & & Berlin & o. Nr. \\
\hline Stern & Naftali & & & Berlin & o. Nr. \\
\hline Sternefeld & Rudolf & & & Berlin & 531 \\
\hline Stössinger & Felix & & & Berlin & 261 \\
\hline Strauß & Bruno & Dr. & Studienrat & Berlin & o. Nr. \\
\hline Strauß & Max & & Rechtsanwalt & Berlin & 243 \\
\hline Strauß & $\operatorname{Max}$ & & & Berlin & 662 \\
\hline Striem & Hans & & & Berlin & 47 \\
\hline Struck & Felix & & Handelsgerichtsrat & Berlin & 12 \\
\hline Theilhaber & Felix A. & Dr. med. & & Berlin & 649 \\
\hline Tietz & Siegfried & & & Berlin & 625 \\
\hline Translateur & Salo & & Direktor & Berlin & 720 \\
\hline Tscherkassky & Joseph & & & Berlin & 409 \\
\hline Tugendreich & Jakob & Dr. med. & & Berlin & 713 \\
\hline Unger & Alfred & & & Berlin & 614 \\
\hline Wagner-Tauber & Lina & & & Berlin & 689 \\
\hline Waldmann & Moses & & & Berlin & 68 \\
\hline Warschauer & Malwin & Dr. & Rabbiner & Berlin & 63 \\
\hline Weigert & Hugo & & & Berlin & 271 \\
\hline Weil & Gotthold & Prof. Dr. & Direktor & Berlin & $71 \mid 72$ \\
\hline Wertheimer & Siegfried & & & Berlin & o. Nr. \\
\hline Wischnitzer & Mark & Dr. phil. & Generalsekretär & Berlin & 241 \\
\hline Wolff & Helene & & & Berlin & 4 \\
\hline Wolff & Siegfried & Dr. jur. & & Berlin & 4 \\
\hline Wollsteiner & Max & & & Berlin & 267 \\
\hline Wunderlich & Gertrud & & & Berlin & 57 \\
\hline Zamory & Gustav & & & Berlin & o. Nr. \\
\hline Zucker & Anni & & & Berlin & 98 \\
\hline Zweig & Arnold & & & Berlin & 9 \\
\hline Goldreich & Max & & & Beuel b. Bonn & 316 \\
\hline Eckstein & Emanuel & Dr. med. & & $\begin{array}{l}\text { Beuthen i. Ober- } \\
\text { schlesien }\end{array}$ & 484 \\
\hline Golinski & Ludwig & Prof. Dr. & Rabbiner & Beuthen i. OS & 510 \\
\hline Jakobowitz & Israel & Dr. & Syndikus & Beuthen i. OS & 207 \\
\hline Jungmann & Arthur & & & Beuthen i. OS & 629 \\
\hline
\end{tabular}




\begin{tabular}{|c|c|c|c|c|c|}
\hline Kunz & Hugo & & & Beuthen i. OS & 628 \\
\hline Wittgenstein & Kurt & & Direktor & Bochum & 542 \\
\hline Heymann & Walther & & & Bottrop & 792 \\
\hline Wilhelm & Kurt & Dr. & Landesrabbiner & Braunschweig & 751 \\
\hline Katzenstein & Julius & Dr. & Rechtsanwalt & Bremen & 313 \\
\hline Markreich & Max & & & Bremen & 291 \\
\hline Bloch & Lippmann & & & Breslau & 332 \\
\hline Engel & Walter & & & Breslau & 150 \\
\hline Hecht & Fritz & & Generaldirektor & Breslau & 730 \\
\hline Jacobsohn & Max & & Rechtsanwalt & Breslau & 622 \\
\hline Laqueur & Ludwig & & & Breslau & 149 \\
\hline Lewin & Leo & & Generaldirektor & Breslau & 617 \\
\hline Mach & Wolfgang & Dipl.-Ing. & & Breslau & 384 \\
\hline Meyer & Franz & Dr. phil. & & Breslau & 290 \\
\hline Polke & Max M. & Dr. jur. & Rechtsanwalt & Breslau & 199 \\
\hline Powitzer & B. & Dr. med. & & Breslau & 700 \\
\hline Prager & Josef & Dr. & & Breslau & 297 \\
\hline Proskauer & Curt & Dr. med. & & Breslau & 283 \\
\hline Schachtel & Hugo & Dr. med. & & Breslau & 84 \\
\hline Unikower & Siegbert & Dr. jur. & Rechtsanwalt & Breslau & 355 \\
\hline Wislicki & Leo & Dr. med. & & Breslau/Berlin & 129 \\
\hline Fleiß & Alfred & Dr. & Referendar & Chemnitz & $23 / 114$ \\
\hline Geis & Fritz & Dr. med. & & Chemnitz & $6 / 44$ \\
\hline Gerson & Walter & & Rechtsanwalt & Chemnitz & $5 / 32$ \\
\hline Holzer & Paul & Dr. med. & & Chemnitz & $48 / 195$ \\
\hline Sichel & Max & Dr. med. & & Chemnitz & 680 \\
\hline Eschelbacher & Siegfried & & & Coblenz & 347 \\
\hline Fröhlich & Elias & Dr. & Rechtsanwalt & Coblenz & 395 \\
\hline Berghold & Georg & & & Danzig & o. Nr. \\
\hline Lichtenstein & Julius Ernst & & Rechtsanwalt & Danzig & 657 \\
\hline Italiener & Bruno & Dr. & Rabbiner & $\begin{array}{l}\text { Darmstadt/ } \\
\text { Hamburg }\end{array}$ & 339 \\
\hline Hirsch & Siegfried & Dr. jur. & Rechtsanwalt & Dresden & 110 \\
\hline Ikenberg & Ludwig & Blas. & & Dresden & 790 \\
\hline Epstein & Harry & Dr. & Rechtsanwalt & Duisburg & 343 \\
\hline Kaufmann & Sally & Dr. & Rechtsanwalt & Duisburg & 521 \\
\hline Lauter & Theodor & & & Duisburg & 543 \\
\hline
\end{tabular}




\begin{tabular}{|c|c|c|c|c|c|}
\hline Strauß & Hermann & & & Duisburg & 545 \\
\hline Traugott & Louis & & Rechtsanwalt & Duisburg & 653 \\
\hline Burger & Norbert & & & Düsseldorf & 781 \\
\hline Flörsheim & Hermann & & & Düsseldorf & $55 / 227$ \\
\hline Franken & Josef & & Landgerichtsrat & Düsseldorf & 461 \\
\hline Gottlieb & Josef & Dr. jur. & Rechtsanwalt & Düsseldorf & 493 \\
\hline Heymann & Fritz & Dr. jur. & & Düsseldorf & $27 / 131$ \\
\hline Ledermann & Martin & & & Düsseldorf & 709 \\
\hline Loewe & Heinrich & Ing. & & Düsseldorf & 258 \\
\hline Stern & Emanuel & & & Düsseldorf & 734 \\
\hline Wisbrun & Walter & Dr. med. & & Düsseldorf & 600 \\
\hline Bauer & Friedrich & Dr. med. & & Elberfeld & $32 / 153$ \\
\hline Blumenthal & Hermann & Dr. med. & & Elberfeld & 603 \\
\hline Goldberg & Richard & & & Elberfeld & $24 / 123$ \\
\hline Schmidt & Robert E. & Dr. med. & Direktor & Elberfeld & 577 \\
\hline Lublinksi & Moritz & & & Elbing/Ostpr. & 99 \\
\hline Weissbrem & Iwan & Dr. med. & & Elbing/Ostpr. & 236 \\
\hline Arndtheim & Artur & & & Erfurt & $53 / 223$ \\
\hline Klein & Ludwig & Dr. & & Erfurt & 327 \\
\hline Wolfheim & Richard & Dr. med. & & Erfurt & 641 \\
\hline Adler & Fritz & $\begin{array}{l}\text { Dr. jur. et Dr. } \\
\text { rer. pol. }\end{array}$ & Syndikus & Frankfurt a.M. & 134 \\
\hline Adler & Josef & & & Frankfurt a.M. & 132 \\
\hline Baer & Edwin & & & Frankfurt a.M. & 34 \\
\hline Baer & Leo & Dr. & & Frankfurt a.M. & 345 \\
\hline Baer & Ludwig & & & Frankfurt a.M. & 590 \\
\hline Bello & Ernst & & & Frankfurt a.M. & 145 \\
\hline Benario & Gustav & & Bankdirektor & $\begin{array}{l}\text { Frankfurt a.M./ } \\
\text { Berlin }\end{array}$ & $\begin{array}{l}412 \mid \\
\text { o. Nr. }\end{array}$ \\
\hline Bier & Max Julius & & & Frankfurt a.M. & 248 \\
\hline Blau & Ernst & Dr. & & Frankfurt a.M. & o. Nr. \\
\hline Bondi & Hugo & & & Frankfurt a.M. & 112 \\
\hline Eisemann & Heinrich & & & Frankfurt a.M. & 105 \\
\hline Ettinghausen & Richard & & & Frankfurt a.M. & 142 \\
\hline Ettlinger & Leo & & & Frankfurt a.M. & 396 \\
\hline Feist & Edwin & & & Frankfurt a.M. & $458 \mid 459$ \\
\hline Freimann & Aron & Prof. Dr. & & Frankfurt a.M. & 24 \\
\hline
\end{tabular}




\begin{tabular}{|c|c|c|c|c|c|}
\hline Frenkel & Max & & & Frankfurt a.M. & 508 \\
\hline Freudenstein & Georg & & & Frankfurt a.M. & 587 \\
\hline Goldschmidt & Leo M. & Dr. jur. & & Frankfurt a.M. & 133 \\
\hline Grünebaum & Michel & Dr. jur. & Rechtsanwalt & Frankfurt a.M. & 596 \\
\hline Heidingsfelder & Ludwig & & & Frankfurt a.M. & 469 \\
\hline Hirsch & Paul & & & Frankfurt a.M. & 546 \\
\hline Hochschild & Moritz & & Direktor & Frankfurt a.M. & 344 \\
\hline Horovitz & Abraham & & Rechtsanwalt & Frankfurt a.M. & 517 \\
\hline Kauffmann & Felix & Dr. phil. & & Frankfurt a.M. & 25 \\
\hline Levy & Max & & Rechtsanwalt & Frankfurt a.M. & 107 \\
\hline Löwenthal & Leo & Dr. & & $\begin{array}{l}\text { Frankfurt a.M./ } \\
\text { Zwickau }\end{array}$ & 123 \\
\hline Mayer & Eugen & Dr. & & Frankfurt a.M. & 122 \\
\hline Miodownik & Ismar & & Bankdirektor & Frankfurt a.M. & 413 \\
\hline Ries & Ludwig & & & Frankfurt a.M. & 151 \\
\hline Rosenberg & Siegfried & & & Frankfurt a.M. & 191 \\
\hline Rosenzweig & Franz & Dr. & & Frankfurt a.M. & $51 / 220$ \\
\hline Rothschild & Henry & & & Frankfurt a.M. & 528 \\
\hline Sänger & Josef & & & Frankfurt a.M. & 302 \\
\hline Schwarzschild & Siegfried & Dr. & & Frankfurt a.M. & 307 \\
\hline Sondheimer & Albert & Dr. & & Frankfurt a.M. & 584 \\
\hline Taubert & Wilhelm & & Kunstmaler & Frankfurt a.M. & 137 \\
\hline Weinberg & Julius & & & $\begin{array}{l}\text { Frankfurt a.M./ } \\
\text { Witten/Emmerich }\end{array}$ & a.o. 219 \\
\hline Werner & Julius & & & Frankfurt a.M. & 119 \\
\hline Weyl & Hermann & Dr. med. & & Frankfurt a.M. & 157 \\
\hline Wolf & Josef & & & Frankfurt a.M. & 575 \\
\hline Zimmer & Carrie & & & Fürth & 765 \\
\hline Mannheimer & Emil & & & Gießen & o. Nr. \\
\hline Kunz & Leonhard & & & Görlitz & 295 \\
\hline Meyer & Andreas & Dr. jur. & Rechtsanwalt & Görlitz & 379 \\
\hline Sommer & Moritz & Dr. & Rechtsanwalt & Görlitz & 414 \\
\hline Warschawski & Fritz & Dr. med. & & Görlitz & 296 \\
\hline Heilbronn & Josef & Dr. med. & & Griesheim & 168 \\
\hline Marxsohn & Ludwig & & & Gross-Gerau & 583 \\
\hline Lichtwitz & Alfred & Dr. med. & & Guben & 449 \\
\hline Zedner & Hans & & Rechtsanwalt & Guben & 178 \\
\hline
\end{tabular}




\begin{tabular}{|c|c|c|c|c|c|}
\hline David & Ferdinand & Dr. & Rechtsanwalt & Hagen i. W. & 378 \\
\hline Leeser & Wilhelm & & & Hagen i. W. & 541 \\
\hline Nassau & Adolf & & Justizrat & Hagen i. W. & o. Nr. \\
\hline Stern & Arthur & & & Hagen i. W./Köln & 591 \\
\hline Hirsch & Menko Max & & & $\begin{array}{l}\text { Halberstadt/ } \\
\text { Berlin }\end{array}$ & 289 \\
\hline Hirsch & Siegfried & & & Halberstadt & 87 \\
\hline Felixbrodt & Marcus & Dr. jur. & Rechtsanwalt & Halle a. S. & 440 \\
\hline Marx & Josef & & & Halle a. S. & 28 \\
\hline Auerbach & Martin & Dr. phil. & & Hamburg & 342 \\
\hline Buchholz & Erich & Dr. & & Hamburg & 315 \\
\hline Cohen & Martin & & & Hamburg & 698 \\
\hline Cohn & Julius & Dr. & & Hamburg & 771 \\
\hline Conitzer & Ludwig & & & Hamburg & 314 \\
\hline David & Bernhard & & Rechtsanwalt & Hamburg & 443 \\
\hline Enoch & Kurt & Dr. & & Hamburg & 456 \\
\hline Fink & Wilhelm & Dr. jur. & Rechtsanwalt & Hamburg & 366 \\
\hline Flörsheim & M. & Dr. jur. & Rechtsanwalt & Hamburg & 431 \\
\hline Frank & Edgar & & & Hamburg & 116 \\
\hline Goetz & Hans & & & Hamburg & 278 \\
\hline Goldschmidt & Fritz & & & Hamburg & a.o. 100 \\
\hline Guggenheim & Wilhelm & & & Hamburg & $9 / 58$ \\
\hline Hayek & Walter & & & Hamburg & 106 \\
\hline Israel & Max & & & Hamburg & $37 / 167$ \\
\hline Koopmann & Alfons & & Bankier & Hamburg & 476 \\
\hline Leßmann & Leo J. & & & Hamburg & 523 \\
\hline Lippmann & Franz & & & Hamburg & 285 \\
\hline Mainz & Hugo & & & Hamburg & 273 \\
\hline Mayer & Heinrich & & & Hamburg & 704 \\
\hline Meyer & Iwan & & & Hamburg & 486 \\
\hline Meyer & Hugo & Dr. & & Hamburg & 756 \\
\hline Offenstadl & Leo & & & Hamburg & 448 \\
\hline Plonsker & Curt & & & Hamburg & 770 \\
\hline Popper & Albert & & & Hamburg & 230 \\
\hline Rosenbacher & Leo & & Direktor & Hamburg & 477 \\
\hline Rosenbaum & Eduard & Dr. phil. & Direktor & Hamburg & 424 \\
\hline Schindler & Irma & & & Hamburg & o. Nr. \\
\hline
\end{tabular}




\begin{tabular}{|c|c|c|c|c|c|}
\hline Schwabe & Fritz & Dr. & & Hamburg & 457 \\
\hline Sender & Benno & Dr. med. & & Hamburg & 620 \\
\hline Warburg & Karl & & & Hamburg & $16 / 96$ \\
\hline Weiß & Samuel & Dr. med. & & Hamburg & $481 \mid 482$ \\
\hline Windesheim & Fritz & & & Hamburg & 776 \\
\hline Stern & Alfred & & & Hanau a. M. & 281 \\
\hline Stern & Kurt & Dr. jur. & & Hanau a. M. & 282 \\
\hline Dammann & Richard & & & Hannover & 606 \\
\hline Schoeps & Georg & Dr. jur. & Rechtsanwalt & Hannover & 300 \\
\hline Bonem & Paul & Dr. med. & & Heidelberg & 172 \\
\hline Deutsch & Saul & & & Heidelberg & 604 \\
\hline Großberger & Herbert & Dr. phil. & & Heidelberg & 317 \\
\hline Hayek & Simon & & & Heidelberg/Berlin & 513 \\
\hline Pinkus & Hermann & Dr. & Rabbiner & Heidelberg & 635 \\
\hline Buber & Martin & Dr. & & Heppenheim & $52 / 221$ \\
\hline Cohn & Leopold A. & Dr. med. & & Hildesheim & 609 \\
\hline Hirsch & Emil & & & Hildesheim & 380 \\
\hline Oppenheimer & Alex. & & $\begin{array}{l}\text { Rechtsanwalt/ } \\
\text { Justizrat }\end{array}$ & Hildesheim & 669 \\
\hline Palmbaum & D. & & & Hildesheim & 670 \\
\hline Rehfeld & Alex & & & Hildesheim & 671 \\
\hline Schönenberger & Paul & & & Hildesheim & 382 \\
\hline Zondervan & Felix & Dr. med. & & Insterburg & 111 \\
\hline Ahlfeld & Ludwig & & & Karlsruhe & 374 \\
\hline Bär & Norbert & Dr. & & Karlsruhe & 203 \\
\hline Bernheimer & Erich & Dr. jur. & & Karlsruhe & 667 \\
\hline Bernheimer & Norbert & Dr.-Ing. & & Karlsruhe & 212 \\
\hline Klopstock & Martin & & & Karlsruhe & 674 \\
\hline Levis & Arthur & Dr. & Rechtsanwalt & Karlsruhe & 786 \\
\hline Marx & Ernst & Dr. & Rechtsanwalt & Karlsruhe & $46 / 186$ \\
\hline Marx & Jakob & Dr. & Rechtsanwalt & Karlsruhe & $45 / 184$ \\
\hline Schiff & Hugo & Dr. & Rabbiner & Karlsruhe & 394 \\
\hline Wolf & Gustav & Prof. & & Karlsruhe & 174 \\
\hline Abraham & Adolf & Dr. med. & & Köln & 550 \\
\hline Bader & Chaim & & & Köln & 165 \\
\hline Bader & Simon & & & Köln & 480 \\
\hline
\end{tabular}


Cahen-Leudes- Hans

Köln

161

dorff

Friedländer Hans

Köln

436

Gidion

Robert

Dr. jur. Rechtsanwalt

Köln

166

Hirschmann Elsbeth

Köln

589

Lissauer Henry

Köln

437

Löser Otto

Köln

322

Marcan

Fritz Jakob

Köln

552

$\mathrm{Ma}(\mathrm{a}) \mathrm{B}$

Alfred

Dr.

Köln

249

Pinette

Max

Dipl.-Ing.

Köln

250

Schiff

Alice

Dr. med.

423

Simchowitz

S.

Dr.

Köln

335

Alexander

Alfred

Dr.

Köln

357

Altmann Leo

Königsberg

398

Cohn

Arthur

Cohn

Edmund

Prof. Dr. med.

Königsberg

399

Königsberg

356

Cohn

Rudolf

Königsberg

320

Davidsohn

Erich

Königsberg

585

Ginsburg

Sigmar

Laser

Isidor

Leß

William

Dr. jur.

Königsberg

231

Rechtsanwalt

Königsberg

400

Königsberg

401

Lewin

Reinhold

Dr.

Rabbiner

Königsberg

402

Loose

Albert

Marx

George

Marx

Hermann

Kommerzienrat

Königsberg

485

Königsberg

96

Nissel

Arthur

Dr.

Bankdirektor

Königsberg

237

Peritz

Adolf

Radziminski Louis

Rosenthal Max

Sabatzky Kurt

Tragheimer Erich Heilbrun

Weißbrem Leonhard

Werblowski Salomon

Wolf

Rahel

Wolkowski

Hermann

Alexander

Felix

Direktor

Königsberg

350

Königsberg 403

Königsberg $\quad 404$

Königsberg 466

Königsberg $\quad 634$

Königsberg 586

Königsberg 14

Königsberg $\quad 405$

Königsberg 235

Königsberg $\quad 406$

Krefeld

229

Bockwitz

Hans $\mathrm{H}$.

Dr.

Leipzig

a.o. 470 


\begin{tabular}{|c|c|c|c|c|c|}
\hline Cohn & Gustav & & Rabbiner & Leipzig & 234 \\
\hline Felsenstein & Alfred & & & Leipzig & 227 \\
\hline Felsenstein & Ernst Moritz & & & Leipzig & 228 \\
\hline Felsenheim & Isidor & & & Leipzig & 228 \\
\hline Hadl & Richard & Prof. & & Leipzig & a.o. 187 \\
\hline Harrassowitz & Otto & & $\begin{array}{l}\text { für Hebrew Union } \\
\text { College Library, } \\
\text { Cincinnati }\end{array}$ & Leipzig & 638 \\
\hline Hiersemann & Karl W. & & & Leipzig & a.o. 186 \\
\hline Jolowitz & Leo & Dr. & & Leipzig & 710 \\
\hline Kaufmann & Willy & Dr. & Rechtsanwalt & Leipzig & 333 \\
\hline Kroch & Fritz & & Direktor & Leipzig & o. Nr. \\
\hline Kroch & Hans & & & Leipzig & o. Nr. \\
\hline Offizin & W. Drugulin & & & Leipzig & a.o. 188 \\
\hline Porges & Oscar & & & Leipzig & 537 \\
\hline Poser & Paul & & & Leipzig & o. Nr. \\
\hline Schick & Rudolf & & & Leipzig & 127 \\
\hline Schub & J. A. & & & Leipzig & 167 \\
\hline Schulz-Besser & Ernst & & & Leipzig & 711 \\
\hline Levy & Siegfried & Dr. med. & & Liegnitz & 441 \\
\hline Nübel & Walter & & & Lippstadt & 525 \\
\hline Steinberg & Curt & Dr. & Assessor & $\begin{array}{l}\text { Lübbecke/ } \\
\text { Castorp }\end{array}$ & 236 \\
\hline Nathan & Louis & & & Magdeburg & 738 \\
\hline Schmulewitz & Philipp & & Bankier & Magdeburg & 213 \\
\hline Simon & Otto & Dr. med. & & Magdeburg & 574 \\
\hline Levi & Sali/Sally & Dr. phil. & Rabbiner & Mainz & 446 \\
\hline Simon & Karl & Dr. med. & & Mainz & 599 \\
\hline Hilb & Karl & Dr. & Rechtsanwalt & Mannheim & 353 \\
\hline Katzenstein & Adolf & Dr. med. & & Mannheim & 156 \\
\hline Kauffmann & Ernst & Dr. phil. & & Mannheim & 126 \\
\hline Kauffmann & Fritz & Dr. med. & & Mannheim & 125 \\
\hline Mansbach & Hermann & Dr. med. & & Mannheim & 131 \\
\hline Simon & Otto & Dr. jur. & Rechtsanwalt & Mannheim & 171 \\
\hline Königsberger & Eduard & Dr. & Rechtsanwalt & Mühlheim a. Ruhr & 592 \\
\hline Auerbach & Heinrich & & & München & 210 \\
\hline Baerwald & Leo & Dr. & Rabbiner & München & 141 \\
\hline Feuchtwanger & Ludwig & Dr. jur. & Rechtsanwalt & München & 365 \\
\hline
\end{tabular}




\begin{tabular}{|c|c|c|c|c|c|}
\hline Feuchtwanger & Sigbert & Dr. & Rechtsanwalt & München & $458 \mid 459$ \\
\hline Feuchtwanger & Theodor & & & München & $47 / 192$ \\
\hline Fraenkel & Alfred & Dr. & & München & 359 \\
\hline Goldberg & Walter & & & $\begin{array}{l}\text { München/ } \\
\text { Plauen }\end{array}$ & 108 \\
\hline Gutmann & Hedwig & & & München & 158 \\
\hline Gutmann & M. J. & Dr. med. & & München & 324 \\
\hline Kirschner & Emanuel & & Oberkantor & München & 491 \\
\hline Lamm & Ignatz & & & München & 226 \\
\hline Lewin & Bruno & & & München & 275 \\
\hline Reich & Jakob & & & München & 646 \\
\hline Rheinstrom & Heinrich & Prof. Dr. & Rechtsanwalt & München & 479 \\
\hline Rieser & Sidney & & & München & 259 \\
\hline Strauß & Eli & Dr. jur. & Rechtsanwalt & München & 225 \\
\hline Strauß & Rafael & Dr. & i.Fa. Holbein-Verlag & München & 223 \\
\hline Weil & Ernst & Dr. phil. & & München & 487 \\
\hline Werner & Alfred & Dr. jur. & Rechtsanwalt & München & 208 \\
\hline Rosenbusch & J. & Dr. med. & & $\begin{array}{l}\text { Naugard i. } \\
\text { Pommern }\end{array}$ & 736 \\
\hline Steinhardt & Georg & Dr. jur. & Rechtsanwalt & Neuwied & 304 \\
\hline Frohnhausen & Paul & Dr. jur. & Rechtsanwalt & Nordhausen & 385 \\
\hline Levy & Alfred & Dr. & Rabbiner & $\begin{array}{l}\text { Nordhausen/ } \\
\text { Bonn }\end{array}$ & 292 \\
\hline Plaut & Berthold & & & Nordhausen & 407 \\
\hline Warburg & Arthur & & Rechtsanwalt & Nordhausen & 376 \\
\hline Warburg & Josef & & & Nordhausen & 293 \\
\hline Warburg & Reinhold & & & Nordhausen & 294 \\
\hline Apfel & Raphael & & & Nürnberg & 501 \\
\hline Baer & Hermann & Dr. med. & & Nürnberg & 352 \\
\hline Bamberger & Isaak & Dr. phil. & & Nürnberg & 215 \\
\hline Bulka & Isaak & & & Nürnberg & 153 \\
\hline Geßner & Hermann & Dr. med. & & Nürnberg & 576 \\
\hline Grünbaum & David & Dr. med. & & Nürnberg & 244 \\
\hline Guggenheimer & Siegfried & Dr. & & Nürnberg & 668 \\
\hline Hichenberg & Frida & & & Nürnberg & $40 / 173$ \\
\hline Kahn & Siegfried & & & Nürnberg & 433 \\
\hline Nußbaum & Meinhold & Dr. & & Nürnberg & 452 \\
\hline Weikersheimer & Isaak & & & Nürnberg & 532 \\
\hline
\end{tabular}




\begin{tabular}{|c|c|c|c|c|c|}
\hline Weil & Max & Dr. med. & & Nürnberg & 351 \\
\hline Guggenheim & Siegfried & Dr. jur. & Rechtsanwalt & Offenbach & 377 \\
\hline Wormser & Arthur & Dr. & Direktor & Offenbach & 49/196 \\
\hline Braunschweiger & David & Dr. & Rabbiner & Oppeln & 245 \\
\hline Kassel & Emil & & & Oppeln & 681 \\
\hline Stein & Gustav & & & Osnabrück & 346 \\
\hline $\begin{array}{l}\text { Herrnstadt- } \\
\text { Oettingen }\end{array}$ & Edith & & & Potsdam-Nowawe & s152 \\
\hline Schreiber & Hermann & Dr. & Rabbiner & Potsdam & $53 \mid 530$ \\
\hline Marcus & Louis & & & Rees a. Rhein & 288 \\
\hline Cohen & Sally & & & Remscheid & 539 \\
\hline Wisbrun & Gustav & & & Remscheid & 540 \\
\hline Stern & Julius M. & & & Rheydt & 747 \\
\hline Nobel & J. & & Rabbiner & $\begin{array}{l}\text { Schneidemühl/ } \\
\text { Berlin }\end{array}$ & 334 \\
\hline Weigersheimer & Moses & & Lehrer & Schweinfurt & 138 \\
\hline Kopfstein & Felix & Dr. jur. & Rechtsanwalt & Seesen & 630 \\
\hline Cohn & Georg & & & Stettin & 319 \\
\hline Dresel & Erich & Dr. & & Stettin & 494 \\
\hline Brotzen & Adolf & & & Stolp i. Pommern & 361 \\
\hline Müllesheim & Fritz & Dr. & Rechtsanwalt & Stolp i. Pommern & 563 \\
\hline Neumann & Max & Dr. med. & & Stolp i. Pommern & 362 \\
\hline Silberstein & Julius & Dr. jur. & Rechtsanwalt & Stolp i. Pommern & 360 \\
\hline Flegenheimer & Eugen & Dr. & Rechtsanwalt & Stuttgart & o. Nr. \\
\hline Hirsch & Otto & Dr. jur. & Ministerialrat & Stuttgart & 143 \\
\hline Krautkopf & Siegfried & & & Stuttgart & 679 \\
\hline Lepmann & Henry & Dr. jur. & Rechtsanwalt & Stuttgart & 467 \\
\hline Posner & Arthur & Dr. & Rabbiner & Stuttgart/Kiel & 135 \\
\hline Rothschild & Oskar & & & Stuttgart & o. Nr. \\
\hline Stern & Ludwig & & $\begin{array}{l}\text { Landgerichts- } \\
\text { direktor }\end{array}$ & Stuttgart & 492 \\
\hline Hermelin & Jakob & & & Ulm & 184 \\
\hline Bamberger & S. & & Rabbiner & Wandsbek & 708 \\
\hline Zeller & Julius & Dr. & Rechtsanwalt & Weilburg & 325 \\
\hline Heimann & Fritz & Dr. & Direktor & Weimar & 495 \\
\hline Blumenthal & Adolf & & & Wiesbaden & 562 \\
\hline Kahn & Walter & Dr. med. & & Wiesbaden & 154 \\
\hline
\end{tabular}




\begin{tabular}{|c|c|c|c|c|c|}
\hline Lazarus & Paul & Dr. & $\begin{array}{l}\text { Stadt- und Bezirks- } \\
\text { rabbiner }\end{array}$ & Wiesbaden & 113 \\
\hline Marxheimer & M. & & $\begin{array}{l}\text { Rechtsanwalt/ } \\
\text { Justizrat }\end{array}$ & Wiesbaden & 568 \\
\hline Staadt & Heinrich & & & Wiesbaden & 276 \\
\hline Eichengrün & Max & & & Witten & 558 \\
\hline Katz & Martha & & & Witten & 554 \\
\hline \multicolumn{2}{|c|}{ Braunschweiger $\mathrm{A}$. } & & Kommerzienrat & Würzburg & 623 \\
\hline Hannover & Siegmund & Dr. & Rabbiner & Würzburg & 354 \\
\hline Lazarus & Ludwig & & & Würzburg & 306 \\
\hline Manasse & Georg & & & Zwickau & 616 \\
\hline Moses & Siegfried & Dr. & Direktor & Zwickau & 140 \\
\hline Noßbaum & Siegfried & Dr. med. & & Zwickau & 420 \\
\hline Schocken & Salman & & & Zwickau & $26 \mid 27$ \\
\hline
\end{tabular}

\section{Weitere Länder}

\section{Belgien}

$\begin{array}{llll}\text { Fink } & \text { Salomon } & \text { Antwerpen } & 103 \mid 104 \\ \text { Jurovics } & \text { Oskar } & \text { Brüssel } & 43\end{array}$

\section{C.S.R. (Tschechoslowakische Republik)}

$\begin{array}{llllll}\text { Brody } & \text { Heinrich } & \text { Dr. } & \text { Oberrabbiner } & \text { Prag } & 665 \\ \text { Eckstein } & \text { Friedrich } & \text { Dr. jur. } & \text { Rechtsanwalt } & \text { Tetschen } & 473 \\ \text { Eisler } & \text { Ernst } & & & \text { Teplitz-Schönau } & 221 \mid 788 \\ \text { Fischer } & \text { Rudolf } & \text { Dr. med. } & \text { Spezialarzt } & \text { Teplitz-Schönau } & 688 \\ \text { Flesch } & \text { Josef } & \text { Dr. phil. } & & \text { Prag } & 159 \\ \text { Goldes } & \text { Josef } & \text { Dr. } & \text { Rechtsanwalt } & \text { Teplitz-Schönau } & 779 \\ \text { Gütig } & \text { Ernst } & \text { Dr. } & \text { Rechtsanwalt } & \text { Prag } & 767 \\ \text { K(n)öpfmacher } & \text { Fritz } & \text { Dr. jur. } & \text { Rechtsanwalt } & \text { Teplitz-Schönau } & 222 \\ \text { Kraus } & \text { Franz } & \text { Dr. } & & \text { Prag } & 768 \\ \text { Laufer } & \text { Hans } & & & \text { Teplitz-Schönau } & 29 / 138 \\ \text { Lederer } & \text { Ernst } & & & \text { Teplitz-Schönau } & 789 \\ \text { Lieben } & \text { S.H. } & \text { Prof. Dr. } & & \text { Prag } & 79 \\ \text { Popper } & \text { Wilhelm } & & & \text { Weisskirchlitz- } & 775 \\ \text { Schick } & \text { Rudolf } & & & \text { Teplitz } & \\ \text { Schleim } & \text { Heinz } & \text { Dr. } & \text { Rechtsanwalt } & \text { Teplitz-Schönau } & 773\end{array}$


Seidemann
Starkenste
Dänemark
Simonsen

David

Prof. Dr.

Kopenhagen

464

Estland

Genß

Julius

Rechtsanwalt Dorpat-Tartu

763

Frankreich

Polak Léon

Dr.

Paris

527

Raphael

Henri

Strasbourg

o. Nr.

\section{Großbritannien}

Elkan Nathan

London

364

Duschinsky

Charles

Dr.

London

564

Gaster

Moses

Dr.

Chacham

London

588

Greenbaum

Josef

Direktor

London

Levy

A. Harold Prof. Dr.

London

749

Samunow

J.

London

613

Weizmann

Chajim

Prof. Dr.

London

$36 / 165$

\section{Italien}

Cheftel

Joseph

Dr. Ing.

Mailand

o. Nr.

Olschki

Leo $S$.

Comm.

Florenz

$54 / 226$

Sonne

Isaia

Prof. Dr.

Florenz

$31 / 152$

\section{Niederlande}

Edersheim

Henri

Dr. jur.

Haag

$15 / 88$

Ehrenfeld Albert

Haag

$20 / 101$

Flörsheim

Salomon

Amsterdam

791

Friedmann $F$.

Hertzberger Menno

Amsterdam

22/106

Hillesum

I.M.

Amsterdam

205

Kleerekoper

A. T.

Amsterdam

251

Koppel

Alfred

Lachmann

Friedrich

Lieme, de

Nehemia

Salomon

Iwan

Amsterdam

772

Rotterdam

800

Haag

$12 / 69$

Direktor

Haag

$11 / 68$

Amsterdam

$1 / 19$ 
Schlössinger Max

Seeligmann Sigmund

Simon

Wam v.

A.

A. S.

\section{Österreich}

Bablik

Baron

Bato

Bromer

Chajes

Diamant

Ebner

Eisler

Fischer

Fleischer

Fleischner

Friedmann

Gelber

Goldhammer

Hirschhorn Sigmund

Hoffmann Norbert

Karbach

Kohn

Krauß

Kreppel

Marg(o)ulies

Präger

Robinsohn

Rothblum

Schöngut

Schwarz

Sommer

Steif

Sträußler

Thorsch

Waldmann

Hans

Salo

Ludwig

Jakob

H.P.

Paul J.

Hermann

Otto

Markus

Max

Ludwig

Marcus

N. M.

Leo

Oskar

Felix

Samuel

Jonas

Isidor

Mayer

Isak

David

Josef

A. $Z$.

Siegmund

Berthold

Heinrich

Emil

Israel
Dr.

Rotterdam

642

Amsterdam

120

Scheveningen

$26 / 125$

Amsterdam

$21 / 104$

Dr. jur. Notar

Prof. Dr.

Wien

190

Dr.

Wien

338

206

Prof. Dr.

Wien

451

Prof. Dr.

Wien

76

Dr.

Oberrabbiner Wien

Wien

504

Dr. jur. Rechtsanwalt Wien

702

Dr.-Ing.

Wien

175

Wien

506

Wien

507

Wien

o. Nr.

Oberrabbiner Wien

509

Dr.

Wien

192

Dr. jur.

Rechtsanwalt Wien

214

Dr. med.

Wien

337

Dr. jur.

Rechtsanwalt

Wien

516

Dr. jur.

Wien

520

Dr. jur.

Rechtsanwalt Wien

522

Prof. Dr.

Wien

336

648

Dr. jur.

Regierungsrat

Wien

434

Dr.

Rechtsanwalt

Wien

238

Dr. med.

Wien

465

Dr.

Wien

778

Ing.

Rechtsanwalt

Wien

170

Dr.

Zivilingenieur

Wien

415

162

164

202

375

Dr.

Wien

605 
Oranje-Freistaat (Südafrika)

Block

M.

Dr.

Bloemfontein

262

\section{Palästina}

Bialik

Ch. N.

Cohn

Heinrich

Cohn

Heinrich

Cohn

Reinhold

Dr. phil.

Goldberg

Isaak

Hoofien

Siegfried

Pflaum

Heinz

Rubaschow

Salman

Zlocisti

Theodor

Maximilian

Majer

Hermann

Bau

Bocko

Mosche

Chajes

Viktor

Chidlowski

Ch.

Goldmann

Bronislaw

Goldstein

Franz

Dr. jur.

Dr.

Prof. Dr.

Rechtsanwalt

Dr.

Direktor

Dr.

Dr. med.
Tel Aviv

Tel Aviv

Jerusalem

Petach-Tikwah

Tel Aviv

Jaffa

Jerusalem

Tel Aviv

Tel Aviv

693
Rabbiner

Lemberg-Lwow

660

Warschau

Zablocie

240

Warschau

690

Lemberg

$0 . \mathrm{Nr}$.

Lodz

Lemberg-Lwow

650

Kattowitz

130

Großkopf Jakob

Hirschfeld

Dr. jur.

Dr.

Stanislaus

Jacubowicz

Jozef

Laski

Louis

Lewin-Epstein Schmul

Dr.

$10 / 59$

658

515

i. Fa. Elias Wielicka

Hirsch Fried-

mann

Reichenstein Marcus

Dr. med.

Prof. Dr.

Dr.

Lodz 703

Warschau

Rabbiner

Kattowitz/Breslau 78

Warschau

Lemberg-Lwow

Warschau

Zloczow/

92

Jerusalem

Krakau

Sobel

Wolf

Dr. phil.

Wilna 


\section{Rumänien}

Rosenwald

Adolf

Cernauti

o. Nr.

Schnapp

Leo

Rusii-Moldovitei

180

Schottland

Sasson

Vera

Kirkcudbright

o. Nr.

\section{Schweiz}

Burstein

Benno

Montreux/Genf

Gunzburg, de Paul Dreyfus

Basel

Spaeth

Arthur

Lugano

S.H.S. (Država Slovenaca, Hrvata i Srba)

$\begin{array}{llllll}\text { Licht } & \text { Alexander } & \text { Dr. jur. } & \text { Rechtsanwalt } & \text { Zagreb } & 719 \\ \text { Rosenberg } & \text { Dragutin } & \text { Dr. } & & \text { Zagreb } & \text { o. Nr. } \\ \text { Tolnauer } & \text { Nikola } & \text { Dr. } & \text { Rechtsanwalt } & \text { Osyek } & 718 \\ \text { Zarkover } & \text { Berthold } & & & \text { Osyek } & \text { o. Nr. }\end{array}$

\section{Ungarn}

Baracs

Karl

Hofrat/Direktor Budapest

663

Blau

Ludwig

Prof. Dr.

Budapest

90

Edelstein

Berthold

Dr.

Oberrabbiner

Budapest

Gebr. Katzburg

Budapest

429

Vereinigte Staaten von Amerika

Bloch

Joshua

Dr.

Blondheim

D.S.

Cohen

Benjamin

Cohen

Davidsohn

S. Solis

Israel

Ember

Aaron

Enelow

H.G.

Friedenwald

Harry

Kormann

Ezra

Loewenberg

Samuel A.

Prof. Dr.

med.

Maisel Max N.

Margolis
Elis

Dr.
New York $\quad 664$

Baltimore

95

759

611

701

270

553

607

612

687

New York

615 


$\begin{array}{lllll}\text { Marx } & \text { Alexander } & \text { Prof. Dr. } & \text { New York } & 86 \\ \text { Rivkind } & \text { Isaak } & & \text { New York } & 538 \\ \text { Schatzky } & \text { Jacob } & \text { Dr. phil. } & \text { New York } & 551 \\ \text { Spivak } & \text { C. D. } & \text { Dr. } & \text { Denver } & 760 \\ \text { Tausner } & \text { M. Ch. } & & \text { New York } & 729 \\ \text { Tscharny-Niger } & \text { Samuel } & & \text { New York } & 632\end{array}$

\section{Bibliotheken und Körperschaften}

\section{C.S.R. (Tschechoslowakische Republik)}

Bibliothek der Israelitischen Gemeinde

Prag $\quad 438$

Bibliothèque Publique et d'Université

Prag

$34 / 157$

\section{Deutschland}

Bayerische Staatsbibliothek

$\begin{array}{ll}\text { München } & \text { a.o. 204 } \\ \text { Halle a. S. } & \text { a.o. 252 } \\ \text { Berlin } & 474 \\ \text { Leipzig } & 246 \\ \text { Berlin } & 60 \\ \text { Berlin } & 682 \\ \text { Danzig } & 266 \\ \text { Hannover } & 652 \\ \text { Köln } & 2 / 20 \\ \text { Königsberg } & 410 \\ \text { Berlin } & 782 \\ \text { Breslau } & 39 / 172 \\ \text { Berlin } & 74 \\ \text { Hamburg } & 303 \\ \text { München } & 224 \\ \text { Leipzig } & \text { a.o. } 242 \\ \text { Berlin } & 94 \\ \text { Eisenach } & 744 \\ \text { Mainz } & 453\end{array}$

Bibliothek der Deutschen Morgenländischen Gesellschaft

Bibliothek der Hochschule für die Wissenschaft des Judentums

Bibliothek der Israelitischen Religionsgemeinde

Bibliothek der Jüdischen Gemeinde

Bibliothek der Jüdischen Reformgemeinde

Bibliothek der Synagogengemeinde

Bibliothek der Synagogengemeinde

Bibliothek der Synagogengemeinde

Bibliothek der Synagogengemeinde

Bibliothek des Central-Vereins deutscher Staatsbürger jüdischen

Glaubens

Bibliothek des jüdisch-theologischen Seminars Fraenckelscher

Stiftung

Bibliothek des Keren Hajessod

Bibliothek Warburg

Cosman-Werner-Bibliothek der Israelitischen Kultusgemeinde

Deutsche Bücherei für „Gesellschaft der Freunde der Deutschen

Bücherei e.V.“

Gesamtarchiv der deutschen Juden

Gesellschaft der Bibliophilen e.V.

Gutenberg-Museum 
Hessische Landesbibliothek

Israelitische Gemeindebibliothek

Israelitisches Gemeindevorsteheramt

Oberrat der Israeliten

Preußische Staatsbibliothek, Orientalische Abteilung

Rabbiner-Seminar

Sächsische Landesbibliothek

Staats- und Universitätsbibliothek Breslau

Staats- und Universitätsbibliothek Hamburg

Stadt-Bibliothek Frankfurt a. M.

Synagogengemeinde Breslau

Synagogenrat Mannheim

Universitäts-Bibliothek Berlin

Universitäts-Bibliothek Leipzig

Vorstand der Israelitischen Gemeinde

Vorstand der Israelitischen Gemeinde

\section{Großbritannien}

Bodleian Library

\section{Niederlande}

Bibliotheca Rosenthaliana

\section{Österreich}

Bibliothek der Israelitischen Kultusgemeinde Jüdisches Museum

Lese- und Redehalle jüdischer Hochschüler

\section{Palästina}

Hebräische National- und Universitätsbibliothek

\section{Polen}

Bibliothek der Großen Synagoge

Bibliothek des Vereins für jüdische Geschichte und Literatur

Bibliotheka i Czytelnia Publiczana „Esra“

\section{Ungarn}

Bibliothek der Landesrabbinerschule

$\begin{array}{ll}\text { Darmstadt } & 608 \\ \text { Nürnberg } & 716 \\ \text { Stuttgart } & \text { o. Nr. } \\ \text { Karlsruhe } & \text { o. Nr. } \\ \text { Berlin } & \text { a.0. } 432 \\ \text { Berlin } & 496 \\ \text { Dresden } & 561 \\ \text { Breslau } & 645 \\ \text { Hamburg } & 35 / 162 \\ \text { Frankfurt a. M. } & \text { a.0. } 218 \\ \text { Breslau } & 672 \\ \text { Mannheim } & 348 \\ \text { Berlin } & \text { a.0. } 386 \\ \text { Leipzig } & 25 / 124 \\ \text { Bremen } & 777 \\ \text { Frankfurt a. M. } & 655\end{array}$

Oxford 557

Amsterdam 82

Wien 798

Wien

Wien

201

Jerusalem

$\begin{array}{ll}\text { Warschau } & 787 \\ \text { Kattowitz } & 257 \\ \text { Krakau } & 146\end{array}$

Budapest 91 
Budai Zsido Közseg - Israelitische Kultusgemeinde

$\begin{array}{ll}\text { Budapest } & 489 \\ \text { Budapest } & 488 \\ \text { Budapest } & 533 \\ \text { Budapest } & 534\end{array}$

O.M.I.K.E. Isr. Landeskultur-Verein

Pester Israelitische Religionsgemeinde

Budapest

534

\section{Vereinigte Staaten von Amerika}

Library of the Jewish Theological Seminary

New York 7

The Dropsie College Broad and York Street

Philadelphia 654

The Hebrew Union College Library

The New York Public Library

Cincinnati

$41 / 175$

New York

602

\section{Logen des U.O.B.B.}

\section{C.S.R. (Tschechoslowakische Republik)}

Großloge für den tschechoslowakischen Staat

Prag

o. $\mathrm{Nr}$.

Loge „Veritas“

Saatz

o. Nr.

Loge Freundschaft

Teplitz-Schönau 793

Loge Moravia

Brünn

o. Nr.

Loge Ostravia

Mähr. Ostrau

$14 / 87$

Loge Union

Pilsen

$19 / 100$

\section{Deutschland}

August-Lamey-Loge
Bibliothek der Berliner Logen
Duisburg-Loge zur Treue
Düsseldorf-Loge
Ferdinand-Gamburg-Loge
Franken-Loge
Frankfurt-Loge
Fraternitas-Loge
Großloge für Deutschland
Hillel-Loge
Jakob-Herz-Loge
Jeremia-Loge
Kaiser Friedrich-Loge

$\begin{array}{ll}\text { Mannheim } & 425 \\ \text { Berlin } & 691 \\ \text { Duisburg } & 28 / 137 \\ \text { Düsseldorf } & 462 \\ \text { Hanau } & 490 \\ \text { Würzburg } & 2 / 176 \\ \text { Frankfurt a. M. } & 49 \\ \text { Dresden } & 298 \\ \text { Berlin } & 439 \\ \text { Hildesheim } & 381 \\ \text { Nürnberg } & 795 \\ \text { Stolp i. } & \\ \text { Pommern } & 363 \\ \text { Bremen } & 372\end{array}$


Lessingloge

Loge zu den drei Erzvätern

Logen-Bibliothek der Kant-Loge

Maimonides-Loge

Makkabi-Loge

Mamreh-Loge

Mendelssohn-Loge

Ostpreußische Loge

Rheinland-Loge

Rhenus-Loge

Silesia-Loge

Steinthal-Loge

Veritas-Loge

Westfalia Loge

Zähringer-Loge

Österreich

Israelitischer Humanitätsverein „Wien“ des Ordens Bnei Brith

\section{Polen}

Izr. Stowaszyszenie humanitarne „Leopolis“ Bnei Brith

Stowarzyszenie Humanitarne-Braterstwo -B'nei-B'rith

$\begin{array}{ll}\text { Breslau } & 471 \\ \text { Tilsit } & 463 \\ \text { Königsberg } & 387 \\ \text { Nürnberg } & 535 \\ \text { Konstanz } & 75 \\ \text { Beuthen i. Ober- } \\ \text { schlesien } & 483 \\ \text { Magdeburg } & 739 \\ \text { Allenstein } & 559 \\ \text { Köln } & 764 \\ \text { Mainz } & 442 \\ \text { Liegnitz } & 417 \\ \text { Hamburg } & 619 \\ \text { Hindenburg i. } & \\ \text { Oberschlesien } & 472 \\ \text { Bielefeld } & 430 \\ \text { Pforzheim } & 388\end{array}$

Wien 478

Lemberg

o. Nr.

Warschau $13 / 85$ 